\title{
A well-balanced discretization for a shallow water inundation model
}

\author{
G. Davies $^{a}$ \\ ${ }^{a}$ Geoscience Australia, GPO BOX 378, Canberra, ACT, 2601 \\ Email: Gareth.Davies@ga.gov.au
}

\begin{abstract}
Hydrodynamic models based on the shallow water equations are commonly used for tsunami, storm surge and flood risk assessment. For a hypothetical inundation event, such models can provide estimates of the extent of inundation, as well as water depth and velocity over time. These outputs may be linked with exposure and vulnerability models to estimate the damage associated with the inundation event, thus providing useful information to inform emergency and risk management.
\end{abstract}

One such model is ANUGA, developed by the Australian National University and Geoscience Australia. ANUGA approximately solves the 2D shallow-water equations on an unstructured mesh using a 'shockcapturing' finite-volume method. This shock-capturing capability is critical for modelling inundation events with rapid changes in flow properties, such as tsunami bores and dam break floods. This is because even in the presence of flow discontinuities, the model can correctly calculate the propagation speed of the discontinuity. Without this shock-capturing capability, the model will generate significant errors in the computation of shock speeds, such as the travel times of dam-break flood waves and steep tsunami.

Mathematically, ANUGA's shock-capturing algorithm requires that terms in the shallow water equations representing the effect of water pressure gradients are split into two components: 1) a term including the influence of the bed slope, and 2) a term including the influence of flow depth gradients. As these two terms are discretized separately, numerical difficulties can arise in situations when they should exactly balance each other (e.g. water at rest in a lake). In some modelled scenarios, these errors can lead to unphysical predictions, such as water 'creeping' up the sides of buildings or wharfs, and high velocities at the edges of stable wet-dry fronts.

Here the implementation of a 'well-balanced' treatment of the pressure gradient in ANUGA is described, that ensures the exact cancellation of the pressure gradient terms when the water surface is flat. This involves the use of an alternative bed-slope discretization and novel adjustments to the treatment of partially wet cells. The adjusted model is able to preserve stationary flow states with complex topography, and shows broad agreement with the original model in a tsunami inundation application.

Keywords: Tsunami, inundation, shallow water equations, finite-volume methods. 


\section{INTRODUCTION}

The two-dimensional shallow water equations are widely used to simulate flows over natural topography, including hydrodynamic disasters such as floods, tsunami and storm surges (e.g. Zoppou and Roberts, 1999; Bradford and Sanders, 2002; Sobey, 2002; Jakeman et al., 2010). They model the time evolution of flow depth and depth-averaged velocity in an incompressible fluid (typically water), in situations where the horizontal length scale of the flow is large compared with the corresponding vertical length scale (e.g. Chaudhry, 2008). They include one equation based on the conservation of flow mass:

$$
\frac{\partial h}{\partial t}+\frac{\partial u h}{\partial x}+\frac{\partial v h}{\partial y}=0
$$

and two equations based on the flow momentum balance:

$$
\begin{aligned}
& \frac{\partial u h}{\partial t}+\frac{\partial\left(u^{2} h+\frac{g}{2} h^{2}\right)}{\partial x}+\frac{\partial u v h}{\partial y}=-g h \frac{\partial z}{\partial x}-g h S_{f x} \\
& \frac{\partial v h}{\partial t}+\frac{\partial u v h}{\partial x}+\frac{\partial\left(v^{2} h+\frac{g}{2} h^{2}\right)}{\partial y}=-g h \frac{\partial z}{\partial y}-g h S_{f y}
\end{aligned}
$$

In Equations 1- 3, $x$ and $y$ are rectangular spatial coordinates in the horizontal plane $(\mathrm{m}), t$ is time (s), $g$ is gravitational acceleration $\left(\mathrm{m} / \mathrm{s}^{2}\right), h$ is the flow depth $(\mathrm{m}), z$ is the bed elevation $(\mathrm{m})$ relative to an arbitrary vertical datum, $u, v$ are the velocities in the $x, y$ directions $(\mathrm{m} / \mathrm{s})$, and $S_{f x}, S_{f y}$ are the 'friction slope' terms in the $x$ and $y$ directions. The latter are usually related to the flow properties via an empirical closure relation. In the present study a Manning friction model is used (e.g. Chaudhry, 2008):

$S_{f x}=n^{2} \frac{\sqrt{u^{2}+v^{2}}}{h^{4 / 3}} u \quad ; \quad S_{f y}=n^{2} \frac{\sqrt{u^{2}+v^{2}}}{h^{4 / 3}} v$

where $n$ is an empirical friction coefficient (termed Manning's $\mathrm{n}$ ), with dimension $\mathrm{s} / \mathrm{m}^{1 / 3}$.

Physically, Equation 1 states that temporal changes in the water depth above any small region of the bed result from differences in the inflows and outflows to that region. Equations 2 and 3 state that the fluid's momentum travels along with the flow, and is simultaneously affected by flow pressure gradients and bed friction. The bed friction acts to dissipate the flow momentum, while the pressure gradient is the key driver of the flow. It provides a source of momentum whenever the stage (i.e. the water surface elevation, denoted $\zeta=z+h$ ) has a non-zero gradient. Conversely, if $\zeta$ is constant, then the pressure gradient is zero, and the flow can reach a stationary state where velocities are zero everywhere (e.g. water at rest in a lake).

\section{NUMERICALLY PRESERVING STATIONARY STATES}

Many numerical algorithms for approximately solving the shallow water equations are not good at representing such 'water at rest' situations, especially if the underlying topography is irregular. Instead, they tend to generate artificial pressure gradients as the stage approaches a constant state. These persistently disturb the flow, and can sometimes lead to significant errors in the model predictions (e.g. Hubbard and Garcia-Navarro, 2000; Sanders et al., 2003; Valiani and Begnudelli, 2006).

An example of this is shown in Figure 1. Here the ANUGA hydrodynamic model (Nielsen et al., 2005) has been used to simulate water with an initially constant free surface elevation $(\zeta=-0.2 \mathrm{~m})$ and zero velocities, in a rectangular basin with a sloping, irregular bed. Although the correct solution should retain this stationary state, the numerical solution generates significant artificial velocities, and small perturbations to the water surface (Figure 1). This is most pronounced near the wet-dry boundary. In practical inundation applications involving near-stationary states, such artificial flows can sometimes noticeably disturb the solution. This may be manifest in unusually high velocities near wet/dry fronts, or small volumes of water 'creeping' into dry regions (Van Drie et al., 2008, Figures 4-6). 

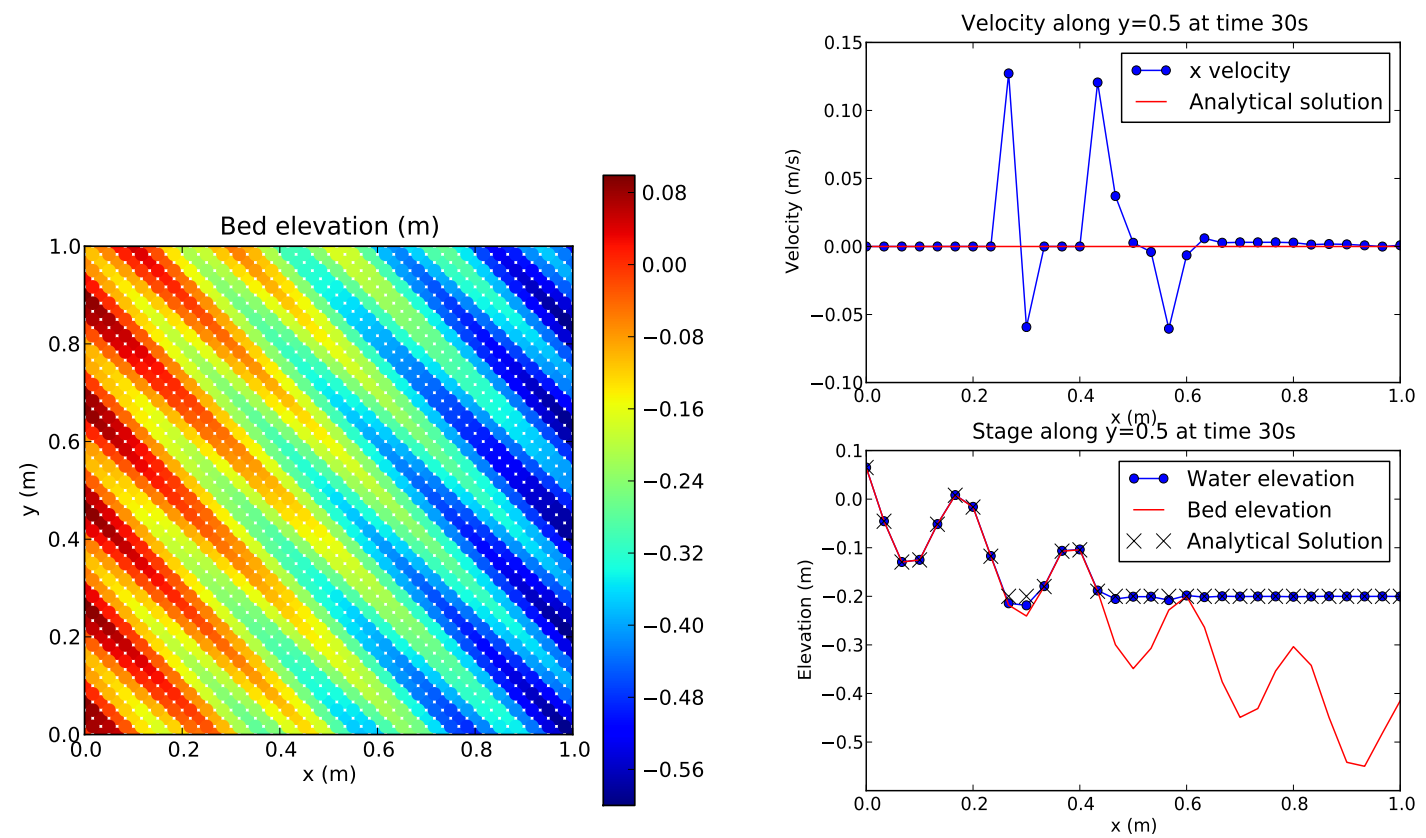

Figure 1: Numerical errors in 'water at rest' with an irregular bed. Left plot shows the bed elevation, and right plots show the computated water velocity and stage at time $30 \mathrm{~s}$, along the line $y=0.5$. The solution should have zero velocities, and a constant stage $(\zeta=-0.2)$ in wet regions of the bed.

Such problems arise because, in Equations 2 and 3, the pressure gradient is represented as the sum of two different terms. In Equation 2 these terms are:

$\frac{\partial}{\partial x}\left(\frac{g}{2} h^{2}\right) \quad \& \quad g h \frac{\partial z}{\partial x}$

and are referred to as the 'depth gradient' and the 'bed slope' term respectively. They sum to give $g h \frac{\partial \zeta}{\partial x}$, which is obviously zero when $\zeta$ is constant in $x$. Analogous statements can be made for Equation 3 using $y$ in place of $x$. Therefore the pressure gradient terms do not influence the flow momentum when $\zeta$ is constant, as expected on physical grounds. However, numerical methods invariably use discrete approximations to represent such derivative terms, which will contain some truncation error. As a result, it is not necessarily true that discrete approximations of the 'depth gradient' and 'bed slope' terms will sum to zero when $\zeta$ is constant, even though the exact equations do have this property. This can cause spurious accelerations in flows near stationary state (Figure 1).

One approach to circumventing this problem is to represent the pressure gradient with a single term in each momentum equation (i.e. $g h \frac{\partial \zeta}{\partial x}$ in Equation 2, $g h \frac{\partial \zeta}{\partial y}$ in Equation 3). As the latter are written directly in terms of stage gradients, it can be easier to ensure that the numerically computed pressure gradient is zero when $\zeta$ is constant. Although this approach is mathematically equivalent to Equations 2 and 3 for smooth flows, it has an important drawback: the pressure gradient terms can no longer be written in 'conservation-law' form, where all terms involving the derivatives of dynamic flow variables $h, u$ and $v$ appear as $\partial F / \partial x$ or $\partial G / \partial y$ (where $F, G$ are functions of the flow variables). For example, the term $\frac{\partial}{\partial x}\left(\frac{g}{2} h^{2}\right)$ is in conservation law form with $F=\frac{g}{2} h^{2}$, while the term $g h \frac{\partial \zeta}{\partial x}$ is not, because of the multiplication by $h$ outside of the derivative term. Numerical methods which are not based on a conservation-law formulation of the mass and momentum equations are expected to incorrectly compute the behaviour of important 'non-smooth' flows, including those caused by dam-breaks, some tsunami, and phenomena such as tidal bores (e.g. Toro, 1999; Stelling and Duinmeijer, 2003; LeVeque, 2004), unless complex shock-tracking procedures are implemented. For this reason, models which aim to correctly simulate such flows usually use the conservation law form of the pressure gradient terms, as in Equations 2 
and 3 .

An alternative solution is to carefully discretize the pressure gradient terms, such that they do cancel when the stage is constant. Several such 'well-balanced' algorithms have been suggested (e.g. Bradford and Sanders, 2002; Valiani and Begnudelli, 2006; George, 2008). A particularly simple and generic approach was suggested by Valiani and Begnudelli (2006). Their method starts from the observation that, when $\zeta$ is constant, it is true that:

$\nabla\left(\frac{g}{2} h^{2}\right)=-g h \nabla z \quad($ when $\zeta=$ constant $)$

where $\nabla=\left(\frac{\partial}{\partial x}, \frac{\partial}{\partial y}\right)$ is the gradient operator. Valiani and Begnudelli (2006) proposed that the numerical value of the terms on the left hand side of Equation 6, evaluated when $\zeta$ is constant, should be used to approximate the terms on the right hand side. This may be applied to compute the 'bed slope' terms even when the stage is not constant, by using a different value of $\zeta$ at each point where the bed slope terms are computed, such that the value of $h$ on the right hand side of Equation 6 is locally correct. With this approach the 'depth gradient' and 'bed slope' terms in Equations 2 and 3 will cancel when the water surface is flat.

\section{IMPLEMENTING A WELL-BALANCED BEDSLOPE DISCRETIZATION IN ANUGA}

The ANUGA hydrodynamic model approximates the solution of the $2 \mathrm{D}$ shallow water equations on an unstructured triangular mesh using an explicit finite-volume method (Nielsen et al., 2005). Essentially, Equations 1-3 are spatially averaged over each mesh triangle. This results in a system of differential equations for the time-evolution of the average depth and momentum in each triangle. The spatial derivatives on the left hand side of Equations 1-3 are then transformed into the sums of the fluxes along the edges of each triangle, using the divergence theorem. To compute these fluxes, the values of $h, u h$ and $v h$ are calculated along the triangle edges, via a piecewise continuous interpolation on each triangle. This leads to two different values of $h, u h$, and $v h$ being computed on each edge. The latter are then used to compute the fluxes across each edge using a Godunov-type method, based on Kurganov et al. (2001).

In ANUGA, the terms on the right hand side of Equations 2-3 are separately discretized, and act as sources of momentum in the spatially averaged versions of Equations 2 and 3. The 'bed-slope' terms are computed for each mesh triangle as:

$g h \nabla z \simeq g h_{c}\left(z_{t x}, z_{t y}\right)$

Here $h_{c}$ is the depth at the centriod of the mesh triangle, and $z_{t x}, z_{t y}$ are the $x$ and $y$ derivatives of the plane connecting the three bed points at the mesh triangle vertices.

This numerical approximation to the 'bed slope' terms does not exactly cancel the numerical approximation of the (spatially averaged) 'depth gradient' terms at stationary state $\left(\zeta=\zeta^{*}=\right.$ constant, $\left.u=v=0\right)$. At stationary state only, ANUGA's discretization of the latter reduces to:

$\frac{1}{A}\left[\iint_{\triangle} \nabla\left(\frac{g}{2} h^{2}\right) d A\right] \simeq \frac{1}{A} \sum_{i=0}^{2} \frac{g}{2}\left(\zeta^{*}-z_{e i}\right)^{2} l_{e i}\left(n_{x}, n_{y}\right)_{e i}$

Here $A$ is the area of the mesh triangle $\triangle, d A$ is an infinitesimal area element, $z_{e i}$ is the bed elevation at the centre of the $i$ 'th edge of the triangle, $l_{e i}$ is the length of the $i$ 'th edge of the triangle, and $\left(n_{x}, n_{y}\right)_{e i}$ is the unit normal vector pointing outward from edge $i$.

Following the method of Valiani and Begnudelli (2006), an alternative discretization of the (spatially averaged) bed-slope term can be developed from Equations 6 and 8:

$\frac{1}{A}\left[\iint_{\triangle} g h \nabla z d A\right] \simeq-\frac{1}{A} \sum_{i=0}^{2} \frac{g}{2}\left(\zeta^{*}-z_{e i}\right)^{2} l_{e i}\left(n_{x}, n_{y}\right)_{e i}$ 
For each mesh triangle, the value of $\zeta^{*}$ is chosen to be equal to the stage at that triangle's centroid. This produces a second order accurate discretization of the bed slope term, since if $z$ and $\zeta$ are linear functions:

$$
\begin{aligned}
& \sum_{i=0}^{2} \frac{g}{2}\left(\zeta^{*}-z_{e i}\right)^{2} l_{e i}\left(n_{x}, n_{y}\right)_{e i} \simeq \int_{\partial \triangle} \frac{g}{2}\left(\zeta^{*}-z\right)^{2}\left(n_{x}, n_{y}\right) d s \\
& =\iint_{\triangle} g\left(\zeta^{*}-z\right) \nabla\left(\zeta^{*}-z\right) d A=-\iint_{\triangle} g(\zeta-z) \nabla z d A
\end{aligned}
$$

Here $\partial \triangle$ denotes the boundary of $\triangle$, and $d s$ is an infinitesimal length element on this boundary.

\subsection{Adjustments for wet/dry boundaries}

If Equation 9 is used to discretize the bed slope term instead of Equation 7, then the numerical method is able to preserve stationary states over complex topography, so long as there are no wet/dry boundaries. However, if dry triangles have wet neighbouring triangles, then further modifications are required.

The reason is that ANUGA does not allow the centroid stage $\zeta_{c}$ in any triangle to fall below the centroid bed elevation $z_{c}$. If this occurs, water is added to the triangle until $\zeta_{c}=z_{c}$. However, $\zeta_{c}$ is typically used to compute the stage at the edges of its triangle. Qualitatively, this can make the model behave as though there is a sharp change in the stage at the shared edge of a wet triangle and a dry triangle, which tends to cause artificial fluxes that prevent the model achieving a stationary state.

For illustration, consider the case of a 'dry' triangle (i.e. $\zeta_{c}^{d}=z_{c}^{d}$, where the $d$ superscript denotes 'dry') which is neighbouring a 'wet' triangle $\left(\zeta_{c}^{w}>z_{c}^{w}\right.$, where the $w$ superscript denotes 'wet'). Suppose that the elevation of their common edge is less than both $\zeta_{c}^{w}$ and $\zeta_{c}^{d}$. At a stationary wet-dry front, we would expect that $\zeta_{c}^{d} \geq \zeta_{c}^{w}$, as otherwise water would flow into the dry triangle. During the flux computation, the values of $\zeta, u h$ and $v h$ must be extrapolated to all edges in both the wet and dry triangles. If a piecewise constant extrapolation is used in both triangles (i.e. $\zeta_{e i}^{d}=\zeta_{c}^{d}, i=0,1,2 ; \quad \zeta_{e i}^{w}=\zeta_{c}^{w}, i=0,1,2$ ), then there will be a 'jump' in the stage at their neighbouring edge. This will generate a flux at this edge (from the 'dry' to the 'wet' triangle) preventing a stationary state from being reached. Similar 'jumps' will occur using other extrapolations of the edge values.

One solution to this, in the case that piecewise constant extrapolation of $\zeta$ is used, is to allow $\zeta_{c}^{d}$ to fall below $z_{c}^{d}$. Instead, it may be bounded below by the minimum bed edge value $\left(\min _{i}\left(z_{e i}^{d}\right)\right)$. This ensures that for all such 'dry' triangles, the depth at all edges is zero, so there can be no outward directed flux.

To faciliate the re-wetting of dry triangles, if $\zeta_{c}<z_{c}$, then the value of $\zeta^{*}$ in Equation 9 is also treated differently. It is computed as the minimum of 1) the average of its stage edge values, as extrapolated from its wet neighbouring triangles, and 2) $\zeta_{c}$. Further, if the term $\left(\zeta^{*}-z_{e i}\right)$ is negative for any edge, then it is set to zero.

\section{EXAMPLES}

\subsection{Water at rest over an irregular bed}

Here the example in Figure 1 is recomputed using the modifications to the ANUGA algorithm described above (Figure 2). The modified model correctly computes the analytical solution, and maintains the stationary state throughout the simulation.

\subsection{Patong tsunami inundation}

Here the inundation of Patong, Thailand during the 2004 Indian Ocean Tsunami is simulated using the ANUGA hydrodynamic model, with and without the modifications described above. This comparison makes use of the freely available data and scripts of Jakeman et al. (2010), which can be consulted for details of the model setup. Figure 3 shows the simulated maximum inundation depth and flow speed around Patong for both algorithms. In the inundation depth plots, only points with a depth greater than $0.01 \mathrm{~m}$ are shown, following Jakeman et al. (2010).

The predictions of both algorithms are similar in many respects. The key differences are the extent of 
inundation in the central portions of the plot. Here the modified algorithm shows less inundation than the unmodified algorithm. In these cases, the unmodified algorithm exhibits broad lobes of relatively shallow inundation (dark blue areas) which are not predicted by the modified model. This may be a result of water 'creep' in the unmodified ANUGA algorithm, although further investigation would be required to confirm this.
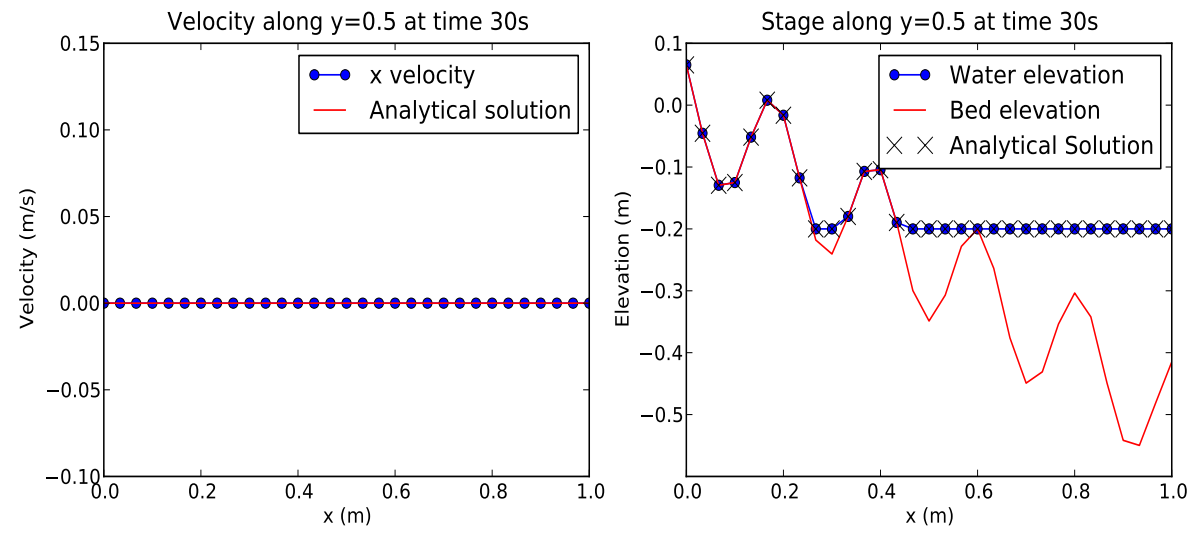

Figure 2: Re-computation of the case in Figure 1 with the modified algorithm. Plots show the computed water velocity (left) and stage (right) at time $30 \mathrm{~s}$, along the line $y=0.5$.
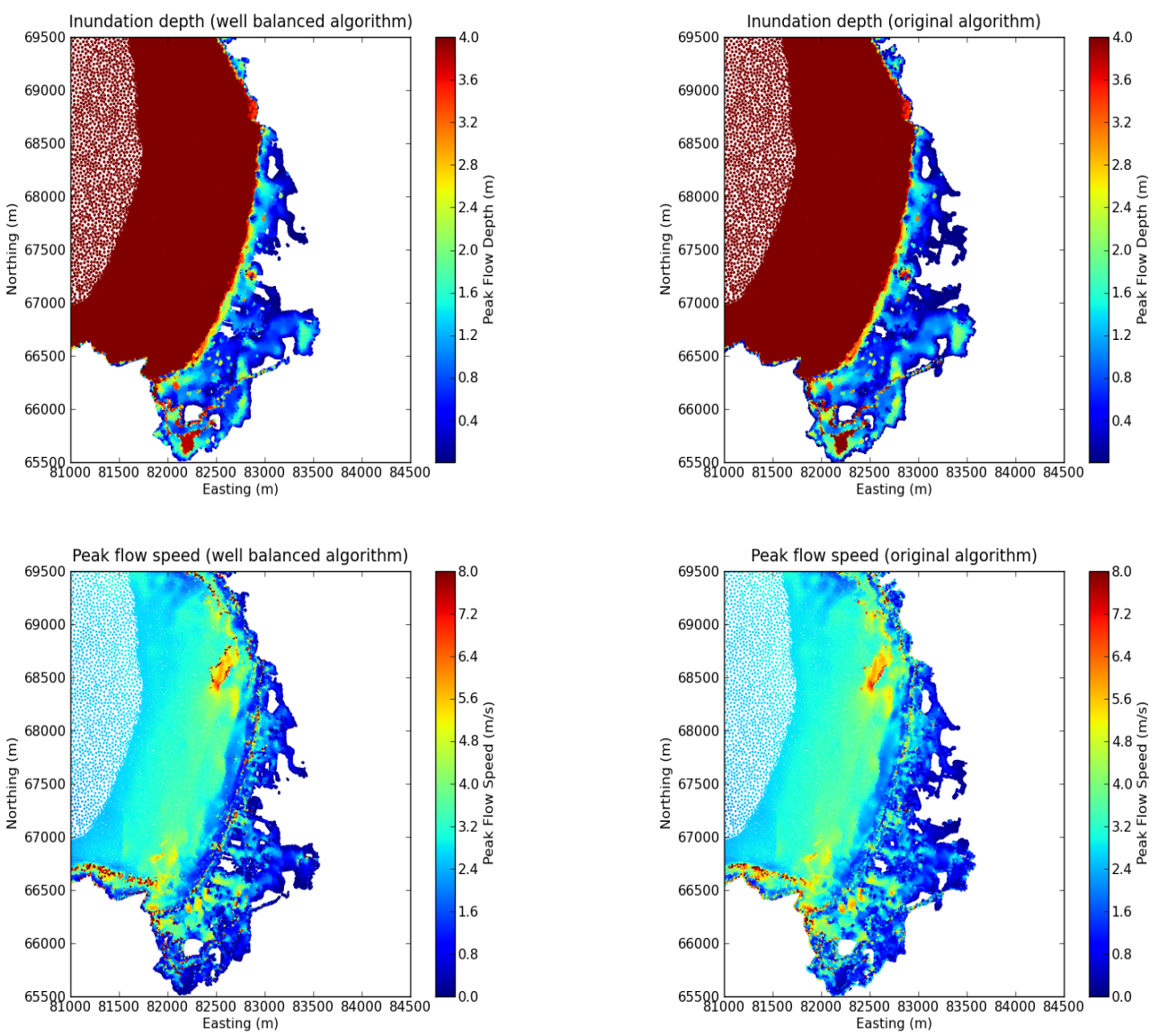

Figure 3: Maximum flow depth and speed during the tsunami at Patong, using the modified ANUGA algorithm (left) and the unmodified ANUGA (right). 


\section{CONCLUSIONS}

A well-balanced discretization of the pressure gradient terms has been implemented in the ANUGA hydrodynamic model. This allows the model to correctly compute stationary state flows, even in the presence of highly irregular bed topographies. Simulations of tsunami inundation at Patong show that the predictions of the well-balanced algorithm are similar to the predictions of the unmodified algorithm, although in the central regions of the township, the inundation extent is smaller in the well balanced algorithm.

\section{REFERENCES}

Bradford, S. F. and B. F. Sanders (2002). Finite-volume model for shallow-water flooding of arbitrary topography. Journal of Hydraulic Engineering 128(3), 289-298.

Chaudhry, M. (2008). Open Channel Flow. New York: Springer.

George, D. L. (2008). Augmented Riemann solvers for the shallow water equations over variable topography with steady states and inundation. Journal of Computational Physics 227, 3089-3113.

Hubbard, M. and P. Garcia-Navarro (2000). Flux difference splitting and the balancing of source terms and flux gradients. Journal of Computational Physics 165, 89-125.

Jakeman, J., O. Nielsen, K. van Putten, R. Mleckzo, D. Burbidge, and N. Horspool (2010). Towards spatially distributed quantitative assessment of tsunami inundation models. Ocean Dynamics 60, 11151138.

Kurganov, A., S. Noelle, and G. Petrova (2001). Semidiscrete central-upwind schemes for hyperbolic conservation laws and Hamilton-Jacobi equations. SIAM Journal on Scientific Computing 23, 707740 .

LeVeque, R. (2004). Finite-Volume Methods for Hyperbolic Problems. Cambridge Texts In Applied Mathematics.

Nielsen, O., S. Roberts, D. Gray, A. McPherson, and A. Hitchman (2005). Hydrodynamic modelling of coastal inundation. In A. Zerger and R. Argent (Eds.), MODSIM 2005 International congress on modelling and simulation., pp. 518-523.

Sanders, B. F., D. A. Jaffe, and A. K. Chu (2003). Discretization of integral equations describing flow in nonprismatic channels with uneven beds. Journal of Hydraulic Engineering 129(3), 235-244.

Sobey, R. J. (2002). Analytical solutions for storm tide codes. Coastal Engineering 46, $213-231$.

Stelling, G. and S. Duinmeijer (2003). A staggered conservative scheme for every froude number in rapidly varied shallow water flows. Internation Journal for Numerical Methods in Fluids 43, 13291354.

Toro, E. (1999). Riemann Solvers and Numerical Methods for Fluid Dynamics. Springer.

Valiani, A. and L. Begnudelli (2006). Divergence form for bed slope source term in shallow water equations. Journal of Hydraulic Engineering 132, 652-665.

Van Drie, R., M. Simon, and I. Schymitzek (2008). 2d Hydraulic modelling over a wide range of applications with ANUGA. In Engineers Australia, 9th National Conference on Hydraulics in Water Engineering, Darwin Convention Centre, Australia 23-26.

Zoppou, C. and S. Roberts (1999). Catastrophic collapse of water supply reservoirs in urban areas. Journal of Hydraulic Engineering 125, 686-695.

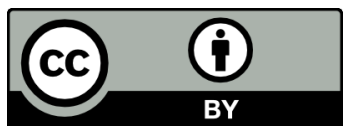

(c) Commonwealth of Australia (Geoscience Australia) 2011.

This material is released under the Creative Commons Attribution 3.0 Australia Licence with the permission of the CEO of Geoscience Australia 